\title{
Combination of Functional Electrical Stimulation and Noninvasive Spinal Cord Electrical Stimulation for Movement Rehabilitation of the Children with Cerebral Palsy
}

\author{
A.G. Baindurashvili, G.A. Ikoeva, Y.P. Gerasimenko, \\ T.R. Moshonkina, I.E. Nikityuk, I.A. Solopova, I.A. Sukhotina, \\ S.V. Vissarionov and D.S. Zhvansky
}

\begin{abstract}
Movement and posture disability are appropriate to cerebral palsy (CP). The aim of the current study was to examine the hypothesis that the functional muscle electrical stimulation (FES) and transcutaneous spinal cord electrical stimulation (TSCS) combined with locomotor treadmill training improve posture and motor function in children with severe $\mathrm{CP}$. Thirty-one children with $\mathrm{CP}$ (spastic
\end{abstract}

\footnotetext{
A.G. Baindurashvili · G.A. Ikoeva · I.E. Nikityuk · S.V. Vissarionov

Turner Pediatric Orthopedic Research Institute, Ministry of Public Health, Moscow, Russia e-mail: turner01@mail.ru

G.A. Ikoeva

e-mail: ikoeva@inbox.ru

I.E. Nikityuk

e-mail: femtotech@mail.ru

S.V. Vissarionov

e-mail: turner01@mail.ru

Y.P. Gerasimenko

Pavlov Institute of Physiology Russian Academy of Sciences, Moscow, Russia

e-mail: yuryg@ucla.edu

T.R. Moshonkina

Pavlov Institute of Physiology Russian Academy of Sciences, LLC Cosyma, Moscow, Russia e-mail: tmoshonkina@gmail.com
}

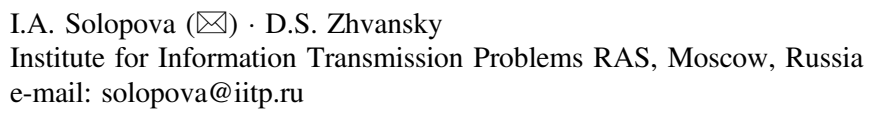

I.A. Sukhotina

First Pavlov State Medical University, Moscow, Russia

e-mail: irina.sukhotina@gmail.com 
diplegia) (7-13 years old; mainly levels III of GMFCS) participated in the study. The experimental group received muscle FES and TSCS at T11 and L1 spinal levels, combined with locomotor treadmill training, whereas the participants of the control group received locomotor treadmill training only. After treatment, the GMFM-88 score increased in $81 \%$ children of the experimental group and in $33 \%$ children of the control group. In the experimental group, there were a significant decrease of the stabilogram area in the eye opened condition and the significant decrease of forward shift of center of pressure projection in the sagittal plane in both eye opened and eye closed conditions, whereas in the control group any significant changes of stabilogram parameters did not observed. Knee torque and range of knee motion significantly increased in the experimental group. After electrical stimulation, the decrease of muscle co-activation in proximal and distal muscles occurred, whereas in the control group muscle co-activation decreased in proximal muscles only. Thus, improvement of motor functions and balance control system in children with severe CP in response to the combination of TSCS, FES and locomotor training revealed. Combination of these techniques can be used for the effective neurorehabilitation.

Keywords Cerebral palsy - Functional electrical stimulation - Transcutaneous spinal cord electrical stimulation $\cdot$ Posture $\cdot$ Rehabilitation

\section{Introduction}

Cerebral palsy $(\mathrm{CP})$ is characterized by muscle weakness, spasticity of extremities, co-activation of antagonistic muscles, resulting in disruption of motor development-pathological stereotype of locomotion, and the impairment of the ability to maintain upright standing $[6,9,26]$. The goal of physical therapy for children with $\mathrm{CP}$ is to promote motor learning through motor and functional training with multiple sensory stimuli.

The main reason of motor impairment - a violation of brain structures, spinal cord changes are secondary. It was suggested that maturation of the spinal locomotor output is impaired in children with CP [4]. Postmortem examination of children with CP revealed abnormalities in motor centers of the brain, brainstem and in the motor nuclei of the cranial nerves as well as in rostral segments of the spinal cord [17]. Magnetic resonance imaging of the spinal cord in the CP subjects with spastic diplegia has significantly less area of the white matter than in healthy individuals in transverse sections at C6/C7 and T10/T11 segments. In the same segments, gray matter area of CP and healthy subjects did not differ [20]. A direct correlation between the degree of imbalance of inhibitory-excitatory connections of motoneurons and the severity of the motor disorders has been reported in $\mathrm{CP}$ subjects [5]. The main problem in regulation of motor functions by spinal neuronal networks is thought to be related to the deficit of supraspinal connections. 
It is known that electrical stimulation applied to spinal cord at L2 spinal segment can induce EMG stepping patterns in leg muscles in spinal cord injured (SCI) patients [7]. We have shown that locomotor training combined with epidural electrical spinal cord stimulation (SCS) facilitated the recovery of voluntary leg movements in motor complete SCI patients [1, 14]. Recently, we developed a method of electrically activating the spinal circuitry via electrodes placed on the skin overlying the lower thoracic vertebrae and demonstrated that in healthy subjects the involuntary locomotor-like movements can be induced by transcutaneous SCS [11, 12]. The positive effect of using transcutaneous SCS (TSCS) was observed in SCI patients as well. It was demonstrated that this neuromodulatory strategy can induce voluntary locomotor-like leg and arm movements after motor complete paralysis [11]. These findings demonstrated that this noninvasive technology can activate spinal neuronal locomotor related networks without brain control in adult chronically paralyzed individuals.

In all cases, SCS induced motor improvement when combined with locomotor training. In recent years, locomotor training on a treadmill widely uses in the treatment of children with CP [16]. Functional electrical stimulation (FES) has been used to enhance the effect of locomotor training [15]. It was shown that in $\mathrm{CP}$ children FES assisted cycling or walking was tolerated well and resulted in increased muscle strength, balance improvement, normalization of biomechanical and innervation structure of locomotor act, increased cadence, power output, and heart rates $[15,19]$.

The aim of the work was to investigate the effect of locomotor training combined with TSCS and FES of legs muscles on postural and locomotor neuronal networks in children with CP. We hypothesized that TSCS combined with FES and locomotor training could be used to recalibrate abnormally developed neuronal networks in children toward a more functional state.

\section{Methods}

Thirty-one children with CP (spastic diplegia) $(9.9 \pm 1.7$ years, mean $\pm \mathrm{SD}$ ) participated in the study (Table 1). All participants previously had surgical interventions to decrease the spasticity and contractures but no later than 2-5 years before the study. The Academic Council of the Turner Scientific and Research Institute for Children's Orthopedics approved this study in accordance with the Declaration of Helsinki. Parents of all participants provided informed consent in writing.

CP participants were mainly level III according to the classification of Gross Motor Function Classification System (GMFCS) [21] and mean leg spasticity $1.5 \pm 0.7$ scores on the Ashworth scale [2]. Children had poor control of vertical posture and were able to keep the upright posture independently for about $2 \mathrm{~min}$. All participants were socialized and able to accurately perform the required tasks. 


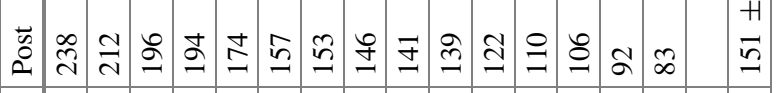

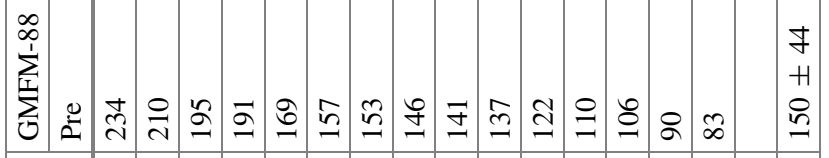

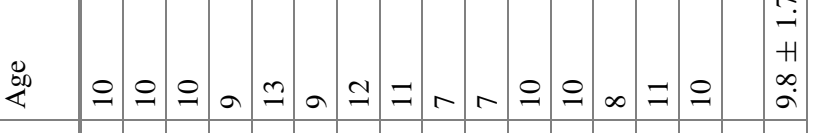

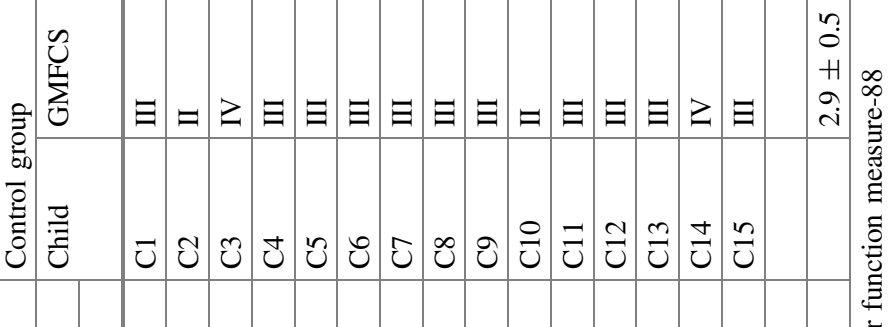

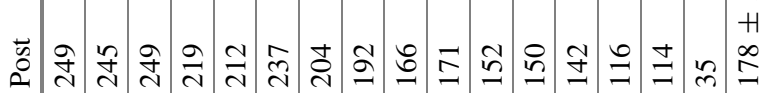

in

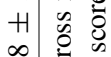

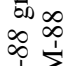

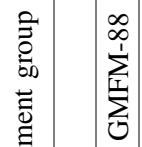

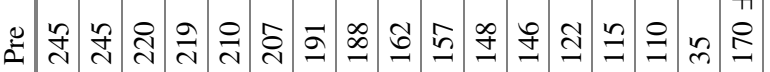

我

站

芉泀

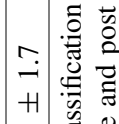

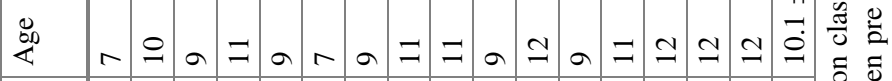

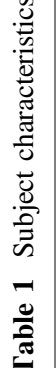

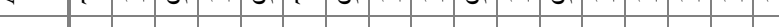

:

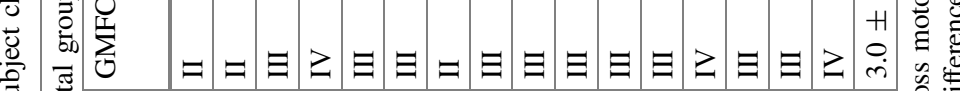

蒠焉

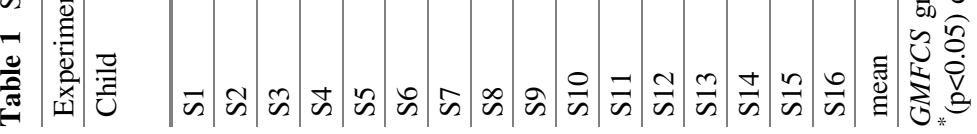


Exclusion criteria were severe contracture of the lower limbs, fractures, osteoporosis, thromboembolic disease, cardiovascular system instability, convulsive readiness, and mental retardation.

Children were randomly assigned to one of two groups (Table 1). The experimental group $(n=16)$ received 15 sessions of locomotor training combined with TSCS and FES over a period of 3 weeks. During each training session, the child was placed in Lokomat device (Hocoma, Switzerland) (Fig. 1a). Game-like augmented performance feedback exercises were used to increase motivation and effort of participants of both groups during treatment and tests. Initially, the TSCS was delivered at L1 level for $5 \mathrm{~min}$ in upright posture. The participants were instructed to keep a normal upright posture. The level of body weight support was selected individually so that the child could stand while maintaining equilibrium. During the first $10 \mathrm{~min}$ of locomotor training, TSCS was applied at T11 vertebral level, followed by the combination of T11 and L1 stimulation for the next $10 \mathrm{~min}$. Afterward, the stepping performance was continued for 20 min with FES of leg muscles assistant. Locomotor training was performed at a treadmill speed of $\sim 1 \mathrm{~km} / \mathrm{h}$. The children of the Control group $(n=15)$ received only locomotor training with Lokomat (40 min) without any electrical stimulation for 15 sessions.

TSCS was delivered using a $2.5-\mathrm{cm}$ round electrodes (Syrtenty ${ }^{\circledR}$, China) placed midline at the T11, and L1 spinous processes as cathodes and two $5.0 \times 8 \mathrm{~cm}^{2}$ rectangular plates (Syrtenty ${ }^{\circledR}$ ) placed symmetrically on the skin over the iliac crests as anodes. Biphasic rectangular $1.0 \mathrm{~ms}$ pulses $(30 \mathrm{~Hz})$, modulated frequency of $10 \mathrm{kHz}$ were used. The main intensity of stimulation ranged from 10 to $50 \mathrm{~mA}$ for most children. Intensity was chosen individually, ranging from 5 to $10 \%$ below threshold of muscle contraction, and was well tolerated by all the participants.

FES method was described in detail previously [19]. A 16-channel stimulator (BiostimES-16, LLC Cosyma, Russia) was used for stimulation (rectangular monophasic pulses; $65 \mathrm{~Hz}$; maximum pulse amplitude $80 \mathrm{~mA}$, pulse width $100 \mu \mathrm{s}$ ). Stimulated muscles were mm longissimus thoracis et illiocostalis, gluteus medius, gluteus maximus, tibialis anterior, quadriceps femoris, extensor digitorum longus et brevis, fibularis longus, extensor hallucis longus et brevis. To accelerate electrode attachment and to prevent electrodes detach while treadmill training as for as for invariance of the position of the electrodes on the body during all procedures, we used a specially designed suit (Fig. 1a) (LLC Cosyma, Russia).

All CP children were tested before and after treatment. Evaluation of the level of motor functions was provided based on GMFM-88 (Gross Motor Function Measure) scale [22]. Calculation of isometric force in flexors and extensors of hip as well as of knee muscles performed using the L-FORCE test of Lokomat software. Evaluation of active range of the movements for flexion/extension in hip and knee joints was performed using the L-ROM test, as directed by Lokomat software. EMG muscle activity (rectus femoris (RF), biceps femoris (BF), lateral gastrocnemius (LG) and tibialis anterior (TA)) was recorded with a telemetric system (MEGA, Finland). EMG activity was tested during stepping on Lokomat belt at the subjects' maximal speed without body weight support, but supported from a horizontal rail, as needed. For assessment of EMG amplitudes, raw EMG signals were 
(a)

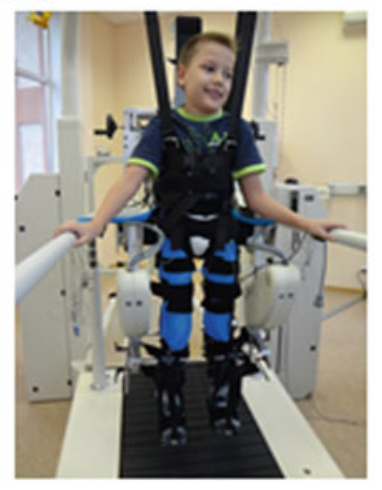

(b)
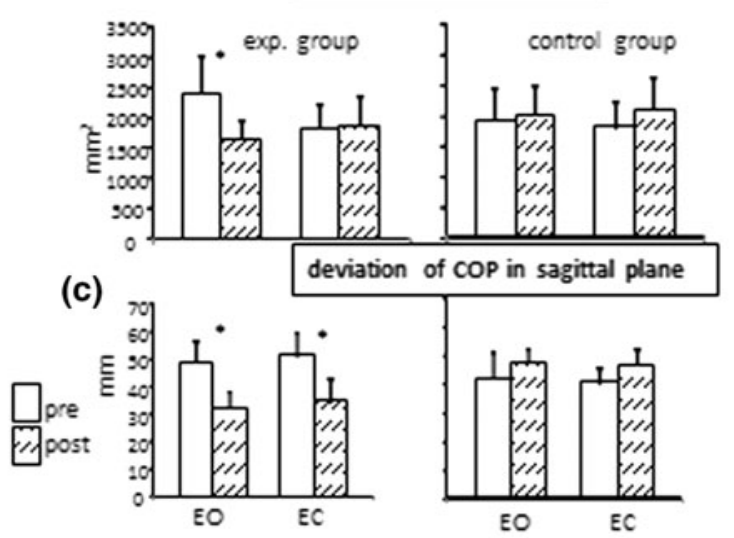

(d)
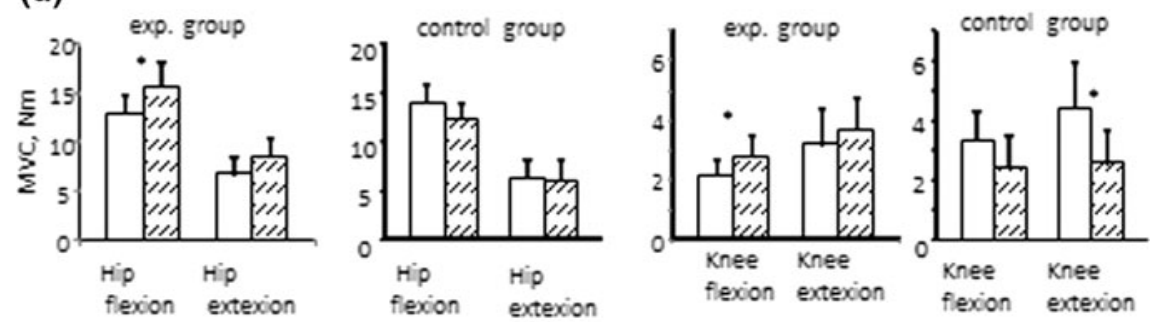

(e)

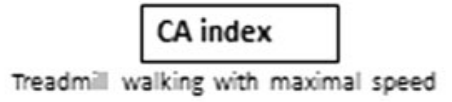

exp. group

control group
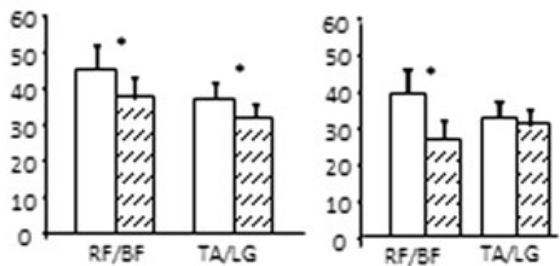

Fig. 1 The changes in stabilogram parameters, Lokomat tests and EMG in participants of both groups before and after treatment. a The participant of experimental group in suit produced by LLC Cosyma before procedure. b The stabilogram area. c Deviation of COP in sagittal plane. d Amplitude of maximal voluntary contraction (MVC) of hip and knee flexors and extensors. e CA values for hip (RF/BF) and ankle (TA/LG) muscle at treadmill walking with maximal speed. Descriptive statistics included means and standard error (SE). Asterisks denote significant differences in the motor functions before and after treatment $(p<0.05)$ 
numerically rectified, low-pass-filtered with a zero-lag Butterworth filter at $20 \mathrm{~Hz}$ cutoff. Co-activation (CA) index of two agonist-antagonist muscle couples (RF/Bf, TA/LG) for both legs were calculated following the method described by Unnithan et al. [27]. Raw EMG data were normalized to the largest value of activation observed in each muscle across all trials for each subject. Integration of the overlapping area between the two normalized linear envelopes defined the CA index. Because participants were diplegic the results for right and left legs were averaged. Lokomat and CA data obtained in control group are the same as have been presented in manuscript "Effects of Spinal Cord Stimulation on motor functions in children with cerebral palsy" [25].

Stabiliometry performed on a "MBN-Biomechanic System" (Moscow, Russia). This system provides measurements of displacement of the center of pressure (COP) along $x$ and $y$ axes in frontal and in sagittal planes, respectively. The assessment was carried out at the standard functional test for $30 \mathrm{~s}$ under each condition of eyes open (EO) and eyes closed (EC). Glasses as needed corrected the vision. The length of COP motion path, area of the stabilogram, deviation of COP along $x$ and $y$ axes were analyzed.

Descriptive statistics included means and standard error (SE). The statistical analysis was performed by Statistica 10 software. The nonparametric MannWhitney U test was used to compare clinical scores and biomechanical values (EMG, L-FORCE, L-ROM, stabilometry parameters) before and after treatment. The Wilcoxon test was performed to compare the same parameters before and after treatment within each group. A level of $p<0.05$ was accepted as statistically significant.

\section{Results}

No advice effects of TSCS or FES were detected. There were no significant group differences in the Ashworth score and GMFCS level (Table 1). Significant increase of the GMFM-88 score was revealed in both groups, but it was significantly higher in the experimental group than in control. The GMFM-88 score increased in 13 of 16 children of the experimental group and in 5 of 15 children of the control group (Table 1).

Initially, the children of experimental and control groups were similar in their ability to maintain upright position. Posture control violation accompanied by greater oscillations of the COP, a substantial increase of length of COP motion path and of the stabilogram area. In all patients, a typical COP forward shift in the sagittal plane was revealed. After treatment in the experimental group, there was a significantly $(p<0.001)$ decrease of area of the stabilogram in the EO condition, whereas in the control group it was not changed in any condition (Fig. 1b). In addition, the decrease of forward shift of COP projection in the sagittal plane in patients of the experimental group in both $\mathrm{EC}(p<0.01)$ and $\mathrm{EO}(p<0.03)$ 
conditions was observed (Fig. 1c). Other analyzed stabilogramm parameters did not change significantly after treatment in both groups.

No differences between groups were found in the knee or hip torque (L-FORCE test) and range of joint movements (L-ROM test) before treatment. After treatment, neither maximal hip flexion nor extension torque changed in the control group (Fig. 1d), but in the experimental group, there was significantly increment in maximal hip flexion torque $(p<0.02)$. Maximal knee flexion and extensor torque increased after electrical stimulation, but this augmentation was significant only for flexion $(p<0.01)$. Unexpectedly in controls flexion and extension knee torques decreased, but significantly for extension $(p<0.05)$ (Fig. 1d). Range of hip movements (L-ROM test) increased in both groups, the change was significant $(p<0.05)$ in control group. In opposite knee range was unchanged in controls (from $17.8 \pm 2.6^{\circ}$ pre to $16.7 \pm 2.7^{\circ}$ post), but it increased in experimental group $(p<0.01)$ (from $13.2 \pm 2.4^{\circ}$ pre to $18.3 \pm 2.9^{\circ}$ post).

The mean treadmill stepping speed before treatment was similar in the experimental and the control groups: $1.4 \pm 0.1$ and $1.3 \pm 0.2 \mathrm{~km} / \mathrm{h}$, respectively. We did not observe significant average speed changes in either group after treatment. Abnormal levels of muscle CA, i.e., showing simultaneous contraction of agonist and antagonist muscles [27], were observed in the experimental and the control groups (Fig. 1e). At the beginning of treatment, there were no differences in CA of either proximal or distal muscles between the experimental and the control group for treadmill stepping. After treatment, the decrease of CA index in proximal and distal muscles occurred in the experimental group, whereas in the control group CA index decreased in proximal muscles only (Fig. 1e).

\section{Discussion}

Previous studies of representative groups of children with $\mathrm{CP}$, with mainly a level III deficit have shown maximal improvement of motor functions up to the age of 10 years with standard rehabilitation including mechanotherapy; afterwards motor skills did not increase and in some cases even decreased [13]. Using the GMFM-88 scale, we have shown that improvement occurred in less than $50 \%$ of the cases in the control group, while in the experimental group $81 \%$ of the subjects showed improved motor skills.

Improvement of balance control was not observed in the Lokomat trained group. Probably the reasons are as severity of disease as pure effectiveness of locomotor training for posture rehabilitation [3]. Opposite after combination of the FES and the TSCS significant positive changes revealed. Center of pressure stabilization and decrease of the pathological body forward inclination were observed. The effect may be connected with the activation of motor pools of muscles involved in maintaining the upright by TSCS [23]. Another reason -increase of strength of many muscles by FES and by this body weight support in upright posture was facilitated [8]. 
Degradation of motor activity in children with CP is linked to with pathology of supraspinal as well as of spinal networks connections during ontogenesis $[17,20]$. One of the functional consequences of the abnormal CP development is the level of abnormal reciprocal inhibition of Ia afferents, presynaptic inhibition and nonreciprocal Ib inhibition a phenomenon largely attributable to spinal networks [10]. We have demonstrated that after TSCS combined with FES and step training the amplitude of movement in both the hip and knee joints increased (Fig. 1d). This observation is consistent with the reduction of co-activation of proximal and distal leg muscles (Fig. 1e). This suggests that TSCS, combined with FES and mechanotherapy, had positive influences on coordination not only for proximal, but for distal muscles as well. Previous studies have shown that improved locomotor performance is closely associated with improved coordination of motor pools [5]. TSCS at T11 and L1 vertebral levels of proximal and distal motor pools in paralyzed subjects are facilitated [24]. This effect is evident in spinal motor evoked potentials of proximal and distal leg muscles to single stimulation pulses [23]. We have found that epidural SCS combined with training can increase hand grip force in spinal patients with cervical injuries [18]. This suggests that similar interventions of training and cervical TSCS could also benefit for children with CP. Our findings of a stable improvement of motor functions and balance control system in children with severe CP in response to the combination of TSCS, FES and locomotor training suggest that some functional improvement of the spinal and supraspinal networks that control locomotor functions were induced. Further studies should receive a high priority.

Acknowledgements The research was conducted with the financial support of the state represented by the Ministry of Education and Science of the Russian Federation. Agreement No 14.576.21.0020 June 27, 2014. Unique project Identifier: RFMEFI57614X0020.

\section{References}

1. Angeli, C.A., Edgerton, V.R., Gerasimenko, Y.P., Harkema, S.J.: Altering spinal cord excitability enables voluntary movements after chronic complete paralysis in humans. Brain 137(5), 1394-1409 (2014)

2. Bohannon, R.W., Smith, M.B.: Interrater reliability of a modified Ashworth scale of muscle spasticity. Phys. Ther. 67(2), 206-207 (1987)

3. Borggraefe, I., Schaefer, J.S., Klaiber, M., et al.: Robotic assisted treadmill therapy improves walking and standing performance in children and adolescents with cerebral palsy. Eur. J. Paediatr. Neurol. 14(6), 496 (2010)

4. Cappellini, G., Ivanenko, Y.P., Martino, G., MacLellan, M.J., Sacco, A., Morelli, D., Lacquaniti, F.: Immature spinal locomotor output in children with cerebral palsy. Front Physiol. 25(7), 478 (2016)

5. Condliffe, E.G., Jeffery, D.T., Emery, D.J., Gorassini, M.A.: Spinal inhibition and motor function in adults with spastic cerebral palsy. J. Physiol. 594(10), 2691-2705 (2016)

6. Damiano, D.L., Martellotta, T.L., Quinlivan, J.M., Abel, M.F.: Deficits in eccentric versus concentric torque in children with spastic cerebral palsy. Med. Sci. Sports Exerc. 33(1), 117$122(2001)$ 
7. Dimitrijevic, M., Gerasimenko, Y., Pinter, M.: Evidence for a spinal central pattern generator in humans. Ann. NY Acad. Sci. 860, 360-376 (1998)

8. Doucet, B.M., Lam, A., Griffin, L.: Neuromuscular electrical stimulation for skeletal muscle function. Yale J. Biol. Med. 85(2), 201-215 (2012)

9. Engsberg, J.R., Ross, S.A., Olree, K.S., Park, T.S.: Ankle spasticity and strength in children with spastic diplegic cerebral palsy. Dev. Med. Child Neurol. 42(1), 42-47 (2000)

10. Filloux, F.M.: Neuropathophysiology of movement disorders in cerebral palsy. J. Child Neurol. 11(1 suppl), S5-S12 (1996)

11. Gerasimenko, Y.P., Lu, D.C., Modaber, M., Zdunowski, S., Gad, P., et al.: Noninvasive reactivation of motor descending control after paralysis. J. Neurotrauma 32(24), 1968-1980 (2015)

12. Gorodnichev, R.M., Pivovarova, E.A., Puhov, A., Moiseev, S.A., Gerasimenko, Y.P., et al.: Transcutaneous electrical stimulation of the spinal cord: a noninvasive tool for the activation of stepping pattern generators in humans. Hum. Physiol. 38(2), 158-167 (2012)

13. Hanna, S.E., Bartlett, D.J., Rivard, L.M., Russell, D.J.: Reference curves for the gross motor function measure: percentiles for clinical description and tracking over time among children with cerebral palsy. Phys. Ther. 88(5), 596-607 (2008)

14. Harkema, S., Gerasimenko, Y., Hodes, J., Burdick, J., Angeli, C., et al.: Effect of epidural stimulation of the lumbosacral spinal cord on voluntary movement, standing, and assisted stepping after motor complete paraplegia: a case study. Lancet 377, 1938-1947 (2011)

15. Harrington, A.T., McRae, C.G., Lee, S.C.: Evaluation of functional electrical stimulation to assist cycling in four adolescents with spastic cerebral palsy. Int. J. Pediatr. 2012, 1 (2012)

16. Johnston, T.E., Watson, K.E., Ross, S.A., et al.: Effects of a supported speed treadmill training exercise program on impairment and function for children with cerebral palsy. Dev. Med. Child. Neurol. 53(8), 742-750 (2011)

17. Levchenkova, V.D., Semenova, K.A.: Contemporary views of the morphological basis of infant cerebral palsy. Zhurnal nevrologii i psikhiatrii imeni SS Korsakova 112(7), 4-8 (2012)

18. Lu, D.C., Edgerton, V.R., Modaber, M., AuYong, N., et al.: Engaging cervical spinal cord networks to reenable volitional control of hand function in tetraplegic patients. Neurorehabil Neural Repair. 30(10), 951-962 (2016)

19. Nikityuk, I.E., Moshonkina, T.R., Shcherbakova, N.A., Vissarionov, S.V., et al.: Effect of locomotor training and functional electrical stimulation on postural function in children with severe cerebral palsy. Hum. Physiol. 42(3), 262-270 (2016)

20. Noble J.J.: Musculoskeletal and Spinal Cord imaging in Bilateral Spastic Cerebral Palsy. Diss. King's College, London (2014)

21. Palisano, R., Rosenbaun, P., Walter, S., Russel, S., Wood, E., Galuppi, B.: Development and reliability of a system to classify gross motor function in children with cerebral palsy. Dev. Med. Child Neurol. 39(4), 214-223 (1997)

22. Russell, D.J., Rosenbaum, P.L., Cadman, D.T., Gowland, C., Hardy, S., Jarvis, S.: The gross motor function measure: a means to evaluate the effects of physical therapy. Dev. Med. Child Neurol. 31(3), 341-352 (1989)

23. Sayenko, D.G., Atkinson, D.A., Dy, C.J., Gurley, K.M., Smith, V.L., et al.: Spinal segment-specific transcutaneous stimulation differentially shapes activation pattern among motor pools in humans. J. Appl. Physiol. 118(11), 1364-1374 (2015)

24. Sayenko, D.G., Atkinson, D.A., Floyd, T.C., Gorodnichev, R.M., Moshonkina, T.R., et al.: Effects of paired transcutaneous electrical stimulation delivered at single and dual sites over lumbosacral spinal cord. Neurosci. Lett. 609, 229-234 (2015)

25. Solopova, I.A., Sukhotina, I.A., Zhvansky, D.S., Ikoeva, G.A., Vissarionov, S.V., Baindurashvili, A.G., Edgerton, V.R., Gerasimenko, Y.P., Moshonkina, T.R.: Effects of spinal cord stimulation on motor functions in children with cerebral palsy. Neurosci. Lett. 639:192-198 (2017)

26. Stackhouse, S.K., Binder-Macleod, S.A., Lee, S.C.: Voluntary muscle activation, contractile properties, and fatigability in children with and without cerebral palsy. Muscle Nerve. 31(5), 594-601 (2005) 
27. Unnithan, V.B., Dowling, J.J., Frost, G., Volpe, Ayub B., Bar-Or, O.: Cocontraction and phasic activity during gait in children with cerebral palsy. Electromyogr. Clin. Neurophysiol. 36, 487-494 (1996)

Open Access This chapter is licensed under the terms of the Creative Commons Attribution 4.0 International License (http://creativecommons.org/licenses/by/4.0/), which permits use, sharing, adaptation, distribution and reproduction in any medium or format, as long as you give appropriate credit to the original author(s) and the source, provide a link to the Creative Commons license and indicate if changes were made.

The images or other third party material in this chapter are included in the chapter's Creative Commons license, unless indicated otherwise in a credit line to the material. If material is not included in the chapter's Creative Commons license and your intended use is not permitted by statutory regulation or exceeds the permitted use, you will need to obtain permission directly from the copyright holder.

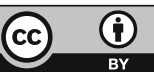

